\section{Gary Bruno Schmid}

\title{
Phantasietherapie in der Behandlung von Psychosen: Vorstellungskraft als Antipsychotikum
}

\author{
Ein Gruppenangebot für stationäre und teilstationäre \\ Patienten in der Allgemeinpsychiatrie
}

- multifunktionellen, d.h. mit einer gleichwertigen Betonung der Funktionen: Denken, Fühlen, Intuition und Verhalten, und

- ontologischen, d.h. erlebnis- und ausdrucksorientierten,

Ansatz für die Behandlung von Psychosen (siehe auch [14]). um eine Gruppentherapie für die Patienten der grossen, öffentlichen psychiatrischen Klinik mit ca. 50\% unfreiwilligen Aufnahmen (FFE: fürsorgerischer Freiheitsentzug) zu entwickeln [2]. Nach positiven Erfahrungen mit diesem Konzept erfolgte die Erweiterung um einen zweiten Teil mit Kunsttherapie (Psychologin Kae Ito sowie die Kunsttherapeuten Stefan Dämpfle und Katharina Frei). Eine positive, evidenzbasierte Qualitätsprüfung der Phantasietherapie 2002/3 $[3,4]$ wurde 2007 durch eine statistische Auswertung der Methode überprüft und bestätigt $[5,6]$. Ein Handbuch für die praktische Anwendung der Phantasietherapie in stationärer und ambulanter Praxis ist in Vorbereitung [7] (für weiterführende Literatur siehe auch [8-13]).

\section{Beschreibung der Methode}

Phantasietherapie utilisiert einen:

- multisensorischen, d.h. Inanspruchnahme von Gleichgewicht (Balance, Bewegung), Gehör-, Geruchs-, Geschmacks-, Seh- und Tastsinn, lich Musik). An dieser 2. Sitzung sind ein/e Psychotherapeut/in und ein/e Kunsttherapeut/in beteiligt.

\section{Zusammenfassung des zweitägigen Ablaufs}

Jede Woche wird ein abstraktes Thema, wie z.B. Energie, Labyrinth, Nähe/ Distanz, Spiegelung, von den Therapeuten ausgewählt (siehe Literatur). Das Thema wird anhand eines Objektes in der Gruppe konkretisiert und diskutiert, vor allem mithilfe von Sprachmetaphern. Anschliessend wird es in Bewegung im weitesten Sinne umgesetzt und mit dem eigenen Körper erfahren und «einverleibt». Hierbei spielen drei Wege der Erfahrung eine zentrale Rolle: die Eigen-, Partner- und Gruppenerfahrung. Schliesslich wird eine Geschichte zum Thema vorgelesen, meistens ein Märchen oder eine Parabel, und das bis dahin Erlebte und Ausgedrückte (Thema, Objekt, Bewegung) wird auf der kognitiv-emotionalen Ebene durchlebt. Am zweiten Tag wird dasselbe Thema durch Wiederholung der Geschichte zu Beginn wieder aufgegriffen und durch die gestalterische Umsetzung in Form und Farbe vertieft.

Die zugrunde liegende Struktur der wöchentlichen Gruppensitzungen von $2 \times 90$ min lässt sich folgendermassen beschreiben [7]:

\section{KARGER}

Fax +497614520714 Information@Karger.de www.karger.com
(๑) 2010 S. Karger GmbH, Freiburg

Accessible online at: www.karger.com/szg
Gary Bruno Schmid, Ph.D

Psychologe/Psychotherapeut SPV/ASP

Trittligasse 2, 8001 Zürich, Schweiz

Tel. +41 442619366

gbschmid@mac.com 
- Erster Tag (1 Psychotherapeut + 1 Bewegungstherapeut): Das gewählte Thema wird

(i) konkret-operationell anhand eines Objekts dargestellt, z.B. eine Art kompliziertes, wissenschaftliches oder pädagogisches Spielzeug,

(ii) ausführlich besprochen, mittels geeigneter Metaphern und Redewendungen, die das Thema ausdrücken,

(iii) kinästhetisch durch Bewegung, Mimik und Gestik über drei zusammenhängende Phasen von Einzel-, Partner- und Gruppenübungen verwandelt und dem Körper sensomotorisch einverleibt,

(iv) sprachlich mittels eines Märchens oder einer Parabel, das den Patienten vom Psychotherapeuten laut vorgelesen wird, symbolisch und kognitivemotional erlebt, währenddem sie ruhig auf Matten mit geschlossenen Augen nach einer meditativen Entspannungsübung liegen. (Beim Vorlesen des Märchens geht die Psychotherapeutin langsam im Raum herum.)

Nach dem Vorlesen des Märchens werden die Patienten nach ihrem bildhaften Erleben gefragt, und dieses wird kurz in der Gruppe diskutiert. Dieser Schritt dient als Brücke zum zweiten Tag unter Leitung eines Kunst- und eines Psychotherapeuten.

- Zweiter Tag (1 Psychotherapeut + 1 Kunsttherapeut): Das gleiche Märchen oder dieselbe Parabel wird nacheinander von den Patienten selbst in der Gruppe laut vorgelesen. Die Patienten werden dann von einem Kunsttherapeuten angeregt, ihre inneren Bilder in Farbe und in Form auszudrücken und eventuell in Symbole zu verwandeln. So wird eine Art Geschichte in Bildern erzählt, die mit dem Thema eng zusammenhängen. Hier kommen vier gestalterische
Strategien zur Geltung: illustrativ, biographisch, abstrakt, absurd [7]. Ein dritter Teil, bei dem die gestalterische Arbeit des zweiten Tages in Rhythmus und Melodie verwandelt wird, ist in Planung.

Tiefenpsychologische Probleme, die während der PhantasietherapieSitzungen aufkommen, werden in darauf folgenden Einzelpsychotherapiestunden behandelt.

Fazit: Die Phantasietherapie ist eine interdisziplinäre Therapieform. Sie integriert Elemente der Bewegungs-, der Kunst- und der sprachlichen Psychotherapie. Eine wöchentliche Thema-Objekt-Märchen-Triade bietet den Patienten einen umfassenden therapeutischen Kontext für jede der zwei (bis drei - siehe oben) Sitzungen einer jeweiligen Woche. Der erste Tag wird gemeinsam von einem Psycho- und einem Bewegungstherapeuten gestaltet, der zweite Tag von einem Psycho- und einem Kunsttherapeuten, der (potenzielle) dritte Tag von einem Psycho- und Musik- bzw. Multimediatherapeuten. (Empfohlen wird, als dritte Ansprechperson für die Patienten einen Therapeuten in Ausbildung bzw. Praktikanten hinzuzunehmen.)

\section{Phantasietherapie im ambulanten Bereich}

Patienten mit remittierendem Krankheitsverlauf oder in ambulanter Behandlung sind für konkrete Themen aus dem Alltag aufnahmebereiter bzw. -fähiger als akutpsychotische Patienten; die Objekte sind vielmals Alltagsgegenstände oder nur selten komplexe Spielzeuge. Sie freuen sich über längere, komplexere Texte (Märchen, Parabel, literarische Anekdoten, Essays, Zeitungsartikel usw.) und begrüssen längere (30-45 anstatt $15 \mathrm{~min}$ ), emotional intensivere Diskussionen des Themas und des Texts. Ambulante Patienten können auch komplexe Handhabungen des Objekts bewältigen, z.B. Jonglieren, und schwierigere
Bewegungsübungen durchführen. Während der letzten Phase der Therapiesitzung (Entspannung durch Atemübungen gefolgt vom Vorlesen des Texts beim Psychotherapeuten) können ambulante Patienten auch tiefere Entspannungs- und Trancezustände erreichen als akut psychotische Patienten.

\section{Personalbedarf}

- 1 Bewegungstherapeut (mit musiktherapeutischen Kompetenzen): Tag 1 ,

- 1 Kunsttherapeut: Tag 2,

- 1 Psychotherapeut: 2 Tage,

- 1 postgraduierter PsychotherapieAssistent (fakultativ): 2 Tage.

\section{Klinische Indikationen}

und Kontraindikationen

Phantasietherapie zielt

- speziell auf die Besserung der Kontextbezogenheit des Patienten, die bei der Psychose durch formale Denkstörungen, Halluzinationen, Wahn und desorganisiertes Verhalten sowie Ambivalenz, Angst, Anhedonie und Autismus gestört ist;

- allgemein auf die Besserung von kognitiven Defiziten: unter anderem Aufmerksamkeits-, Konzentrations-, Kurzzeitgedächtnis-, Planungs-/Problemlösungsstörungen und Störungen im strategischen Denken.

Die speziellen Ziele betreffen Patienten, die unter einer psychotischen Störung leiden, vor allem unter Schizophrenie (ICD-10 F2). Die allgemeinen Ziele schliessen praktisch alle anderen Patienten ein. Bis jetzt haben wir keine klinische Kontraindikation für die Phantasietherapie gefunden. Es hat sich aber herausgestellt, dass Patienten, die Substanzen missbrauchen (ICD-10 F1), diese spielerische Form der Therapie oft zu kindisch finden, d.h. sie haben im Allgemeinen 
einen starken unbewussten Widerstand, an der Gruppenidentität teilzunehmen.

\section{Abweichungen von den Mainstream-Methoden}

Die Phantasietherapie bietet den $\mathrm{Pa}$ tienten auf eine humorvolle und spielerische Art und Weise eine schöpferische Freiheit innerhalb einer therapeutischen Geborgenheit (= das japanische "Amae-Prinzip» $[16,17])$. Sie stellt auch einige klassische Vorbehalte gegenüber bestimmten Behandlungsweisen von akut psychotischen Menschen infrage:

- Gruppentherapie

- grosse Gruppen

- Nähe und Berührung

- multisensorische Erlebnis- und Ausdrucksarbeit

- phantasievolle Vorstellungsarbeit einschliesslich der Arbeit mit Märchen

- Tabu-Themen mit emotional hoch beladenem Inhalt (z.B. Märchen)

- Wirksamkeit mit nur einer einzigen Sitzung.

\section{Qualitätssicherung}

Deskriptive statistische Patientenerhebungen während der Entwicklungsphase der Phantasietherapie ge- ben Hinweise darauf, dass die sog. WZW-Kriterien des Schweizerischen Bundesamtes für Gesundheitswesen von dieser Therapieform erfüllt werden $[1,4,5]$ :

- Wirksamkeit (!AJA!-Effekt und Abschwächung der Desorganisiertheit und des Aggressionspegels in ein oder zwei Sitzungen à $90 \mathrm{~min}$ ).

- Zweckmässigkeit (Eignung für fast alle Diagnosegruppen, insbesondere für Psychosen; Eignung für alle Schweregrade der Störungen; Motivation aggressiver, negativistischer und antriebsgestörter Patienten).

- Wirtschaftlichkeit (bis zu 18 Patienten $/ 3$ Therapeuten $\times 1,5 \mathrm{~h}=$ bis $\mathrm{zu}$ 9Patienten-StundenproTherapeut).

\section{Literatur}

1 Schmid GB, Eisenhut R, Dämpfle S, Frei K, Ito K: Phantasietherapie: In der Phantasie die Realität wieder finden. Tandem 1997;2:21-23.

2 Schmid GB, Eisenhut R, Rausch A, Ito K, Dämpfle S, Frei K, Giacometti Bickel G: Phantasietherapie: In der Phantasie die Realität wieder finden. «Das praktische Gerüst». Forum für Kunsttherapie 2000;2:34-49.

3 Schmid GB, Eisenhut R, Rausch A, Ito K, Dämpfle $S$, Frei K: Phantasy therapy in psychiatry: rediscovering reality in phantasy. A special treatment for in- and outpatients in general psychiatry. Forsch Komplementarmed Klass Naturheilkd 2002;9:283-291.

4 Hänni S: Eine Untersuchung der punktuellen Nützlichkeit der Fantasietherapie: Eine Gruppentherapie für stationäre psychotische $\mathrm{Pa}$ tienten. Unveröffentlichte Diplomarbeit. Zürich, Hochschule für Angewandte Psychologie HAP, 2003.
5 Schmid GB, Wanderer S: Phantasy therapy: statistical evaluation of a new approach to group psychotherapy for stationary and ambulatory psychotic patients. Forsch Komplementmed 2007;14:216-223.

6 Schmid GB, Brunisholz K: Evaluation der Inanspruchnahme komplementärmedizinischer Heilverfahren bei schizophrenen PatientInnen. Forsch Komplementmed 2007;14:167-172.

7 Schmid GB, Ito K, Eisenhut Müller R: Phantasietherapie: In der Phantasie die Realität wieder finden. Wien, Springer, 2011.

8 Schmid GB: Die Bedeutung Benedettis für die Therapie der Schizophrenie. Zu Ehren von Gaetano Benedetti. Forum für Kunsttherapie 2001;1/2:3-19

9 Schmid GB: Die Bedeutung Benedettis für die Therapie der Schizophrenie. Zu Ehren von Gaetano Benedetti. CH-Hypnose Bulletin 2002;XII:4-22.

10 Schmid GB: Phantasy Therapy: a novel theoretic and therapeutic approach for the special treatment of psychotic patients in general psychiatry; in Abelian ME (ed): Focus on Psychotherapy Research. New York, Nova Science, 2005, pp 1-50.

11 Schmid GB: Phantasy therapy: use of story in group psychotherapy. Psychiatric Times 2005;XXII:68-74.

12 Schmid GB: Selbstheilung durch Vorstellungskraft, ed 1. Wien, Springer, 2001.

13 Eisenhut Müller R: Der multimodale Ansatz in der Therapie: Ein Literaturvergleich. Unveröffentlichte Diplomarbeit. Zürich, Hochschule für Heilpädagogik, 2008.

14 Zimmer R: Handbuch der Sinneswahrnehmung: Grundlagen einer ganzheitlichen Bildung und Erziehung, ed 16. Freiburg i.Br., Herder, 2005.

15 Schmid GB: Biunity (Îkilibirlik). Ankara, Agarta Yayinlari, 2008.

16 Doi T: Amae: Freiheit in Geborgenheit: Zur Struktur japanischer Psyche. Frankfurt/M., Suhrkamp, 1982.

17 Ito K: Amae-Psychologie: Ein japanischer Beitrag zur Psychoanalyse. Asiatische Studien 1994;XLVIII:1331-1336. 